\title{
Preoperative stereotactic radiosurgery before planned resection of brain metastases: updated analysis of efficacy and toxicity of a novel treatment paradigm
}

\author{
Roshan S. Prabhu, MD, MS, ${ }^{1,2}$ Katherine R. Miller, BS, ${ }^{1}$ Anthony L. Asher, MD, ${ }^{1,3}$ \\ John H. Heinzerling, MD, ${ }^{1,2}$ Benjamin J. Moeller, MD, PhD, ${ }^{1,2}$ Scott P. Lankford, MD, ${ }^{1,2}$ \\ Robert J. McCammon, MD, ${ }^{1,2}$ Carolina E. Fasola, MD, MPH, ${ }^{1,2}$ Kirtesh R. Patel, MD, ${ }^{4}$ \\ Robert H. Press, MD, ${ }^{5}$ Ashley L. Sumrall, MD, ${ }^{1}$ Matthew C. Ward, MD, ${ }^{1,2}$ and Stuart H. Burri, MD ${ }^{1,2}$ \\ ${ }^{1}$ Levine Cancer Institute, Atrium Health; ${ }^{2}$ Southeast Radiation Oncology Group; and ${ }^{3}$ Carolina Neurosurgical and Spine \\ Associates, Charlotte, North Carolina; ${ }^{4}$ Department of Therapeutic Radiology, Yale University School of Medicine, New Haven, \\ Connecticut; and ${ }^{5}$ Department of Radiation Oncology, Emory University and Winship Cancer Institute, Atlanta, Georgia
}

OBJECTIVE Preoperative stereotactic radiosurgery (SRS) is a feasible alternative to postoperative SRS and may lower the risk of radiation necrosis (RN) and leptomeningeal disease (LMD) recurrence. The study goal was to report the efficacy and toxicity of preoperative SRS in an expanded patient cohort with longer follow-up period relative to prior reports. METHODS The records for patients with brain metastases treated with preoperative SRS and planned resection were reviewed. Patients with classically radiosensitive tumors, planned adjuvant whole brain radiotherapy, or no cranial imaging at least 1 month after surgery were excluded. Preoperative SRS dose was based on lesion size and was reduced approximately 10-20\% from standard dosing. Surgery generally followed within 48 hours.

RESULTS The study cohort consisted of 117 patients with 125 lesions treated with single-fraction preoperative SRS and planned resection. Of the 117 patients, 24 patients were enrolled in an initial prospective trial; the remaining 93 cases were consecutively treated patients who were retrospectively reviewed. Most patients had a single brain metastasis (70.1\%); $42.7 \%$ had non-small cell lung cancer, $18.8 \%$ had breast cancer, $15.4 \%$ had melanoma, and $11.1 \%$ had renal cell carcinoma. Gross total resection was performed in $95.2 \%$ of lesions. The median time from SRS to surgery was 2 days, the median SRS dose was $15 \mathrm{~Gy}$, and the median gross tumor volume was $8.3 \mathrm{~cm}^{3}$. Event cumulative incidence at 2 years was as follows: cavity local recurrence (LR), $25.1 \%$; distant brain failure, $60.2 \%$; LMD, $4.3 \%$; and symptomatic RN, 4.8\%. The median overall survival (OS) and 2-year OS rate were 17.2 months and $36.7 \%$, respectively. Subtotal resection $(S T R, n=6)$ was significantly associated with increased risk of cavity LR (hazard ratio [HR] 6.67, $p=0.008$ ) and worsened OS (HR 2.63, $p=0.05)$ in multivariable analyses.

CONCLUSIONS This expanded and updated analysis confirms that single-fraction preoperative SRS confers excellent cavity local control with very low risk of RN or LMD. Preoperative SRS has several potential advantages compared to postoperative SRS, including reduced risk of RN due to smaller irradiated volume without need for cavity margin expansion and reduced risk of LMD due to sterilization of tumor cells prior to spillage at the time of surgery. Subtotal resection, though infrequent, is associated with significantly worse cavity LR and OS. Based on these results, a randomized trial of preoperative versus postoperative SRS is being designed.

https://thejns.org/doi/abs/10.3171/2018.7.JNS181293

KEYWORDS stereotactic radiosurgery; brain metastases; leptomeningeal disease; neurosurgery; radiotherapy; oncology

$\mathrm{P}$ ATIENTS with solid cancers have a brain metastasis incidence of up to $30 \%$, and these metastatic lesions are a significant source of morbidity and mortality. ${ }^{18}$ Lung, breast, and melanoma primaries are the most associated with brain metastasis development and account for
$67 \%-80 \%$ brain metastasis cases. The reported incidence of brain metastasis has increased in recent years, possibly due to improved sensitivity of neuroimaging, improved systemic therapies with limited brain penetration, or a combination of factors. ${ }^{23}$

ABBREVIATIONS DBF = distant brain failure; $\mathrm{GPA}=$ graded prognostic index; $\mathrm{GTR}=$ gross-total resection; $\mathrm{GTV}=$ gross tumor volume; $\mathrm{HR}=$ hazard ratio; $I Q R=$ interquartile range; $L M D=$ leptomeningeal disease; $L R=$ local recurrence; $N S C L C=$ non-small cell lung cancer; $O S=$ overall survival; $R C C=$ renal cell carcinoma; $R N=$ radiation necrosis; RT = radiotherapy; SRS = stereotactic radiosurgery; STR = subtotal resection; WBRT = whole-brain RT.

SUBMITTED May 10, 2018. ACCEPTED July 16, 2018.

INCLUDE WHEN CITING Published online December 14, 2018; DOI: 10.3171/2018.7.JNS181293. 
Stereotactic radiosurgery (SRS) has become the preferred therapy for patients with a limited number of brain metastases (defined as 1-4) based on multiple Phase III trials demonstrating significant reduction in the risk of neurocognitive and quality-of-life decline and no detriment in overall survival (OS) with the omission of wholebrain radiotherapy (WBRT). ${ }^{1,3,8,9,14,24}$ In the setting in which resection of brain metastasis is performed, resection alone has an expected 1-2 year local recurrence (LR) rate of 47\%-59\%; hence radiotherapy (RT) as an adjunct to resection is generally recommended to reduce risk of cavity LR. ${ }^{14,16,19}$

Two recently published Phase III trials have demonstrated postoperative SRS as a potential standard of care after resection of brain metastases based on improved cavity LR rates compared with surgery alone and reduced neurocognitive deterioration compared with postoperative WBRT, $^{7,16}$ There has also been increasing evidence that patients treated with postoperative SRS have an increased incidence of leptomeningeal disease (LMD) recurrence, with rates of up to $30 \%$.

Our group has previously demonstrated the feasibility of preoperative SRS given as a single fraction prior to planned resection in a cohort of 47 patients. ${ }^{2}$ We have also shown that preoperative SRS (in a cohort of 66 patients) has the potential to reduce the risk of radiation necrosis (RN) and LMD recurrence with similar cavity LR rates compared with postoperative SRS..$^{20}$ The goal of this study was to determine the efficacy and toxicity of preoperative SRS in an expanded patient cohort with longer imaging and clinical follow-up period.

\section{Methods \\ Patients}

Patient treated at the Levine Cancer Institute, Atrium Health (Charlotte, North Carolina) between 2005 and 2016 were eligible for inclusion in this study. The records for patients with brain metastases treated with preoperative SRS and planned resection were reviewed. Patients with classically radiosensitive tumors (small cell carcinoma, lymphoma, germ cell tumor, etc.), planned adjuvant WBRT, previous WBRT, or no cranial imaging at least 1 month after surgery were excluded. Institutional review board approval was obtained from Chesapeake IRB for this study.

\section{Treatments}

The treating neurosurgeon made the decision for resection based on tumor location, associated symptoms, tumor size, and need for tissue analysis. Patients requiring immediate or urgent surgical decompression underwent initial resection and were considered for postoperative SRS and were not included in this study cohort. Patients who would benefit from resection and had improving or stable symptoms with steroid treatment were considered for preoperative SRS. Neurosurgical resection was then performed according to standard technique using an MRI-based surgical navigation system, with gross-total resection (GTR) achieved whenever possible. The details of preoperative SRS planning, delivery, and timing have been previously published.,20,21 In brief, preoperative SRS was delivered as a single fraction using a linear accelerator with a 6-MV photon beam and a frameless radiosurgery technique using the Novalis Tx system (Varian) or Cyberknife system (Accuray Inc.). The planning target volume (PTV) was defined as the enhancing brain metastasis with no additional margin (gross tumor volume $[\mathrm{GTV}]=\mathrm{PTV}$ ). The radiation dose was reduced by approximately $10-20 \%$ compared with standard dosing and was based on lesion volume (Fig. 1). ${ }^{22}$ Preoperative SRS was generally delivered within 48 hours of planned surgery.

\section{Follow-Up}

Patients were evaluated with clinical examination and MRI of the brain with and without a contrast agent 6 to 8 weeks after resection. Patients were then followed with regular clinical examinations and brain MRI every 3-4 months thereafter, unless follow-up was clinically indicated at an earlier time point.

\section{Statistical Analyses}

The date of resection was considered time 0 for timeto-event analyses. Local recurrence (LR) was defined as the presence of new, progressive nodular enhancement involving the resection cavity seen on contrast-enhanced MRI. Radiation necrosis (RN) was defined on the basis of 2 radiological criteria: 1) the development of a contrastenhancing mass within previous radiation treatment fields and 2) conventional imaging features, such as "soap bubble" appearance..$^{15}$ If there was a question of the enhancement representing LR or RN, additional advanced imaging (e.g., MR perfusion, MR spectroscopy, or brain positron emission tomography) was performed and consensus was reached in a multidisciplinary neuro-oncology tumor board. If the patient underwent surgery and the pathology showed any viable cancer, the event was coded as an LR. Distant brain failure (DBF) was defined as the presence of 1 or more new enhancing lesions $\geq 5 \mathrm{~mm}$ away from the treated prescription isodose line. For patients with multiple lesions, recurrence or progression at unresected sites was considered DBF. Leptomeningeal disease (LMD) was defined as new, abnormal leptomeningeal enhancement consistent with malignant leptomeningeal involvement $\geq 5 \mathrm{~mm}$ away from the treated prescription isodose line. Intracranial event rates were estimated using the cumulative incidence with competing risk of death methodology. Patients were censored at time of last brain imaging or time of salvage WBRT (if performed for reasons other than the event of interest, e.g., in the LR analysis if salvage WBRT was given for DBF without evidence of cavity LR). Patients were censored at time that they were last known alive for the salvage WBRT analysis. The composite endpoint of cavity LR, symptomatic RN, or LMD was evaluated to assess a measure of the overall efficacy and toxicity of this therapy using the same method as previously described. ${ }^{20}$ Time 0 was date of resection, and the event was the first incidence of cavity LR, symptomatic RN, or LMD. Patients without an event were censored at time of last brain imaging. Multivariable analysis was performed using the method of Fine and Gray. ${ }^{11}$ The event for OS 


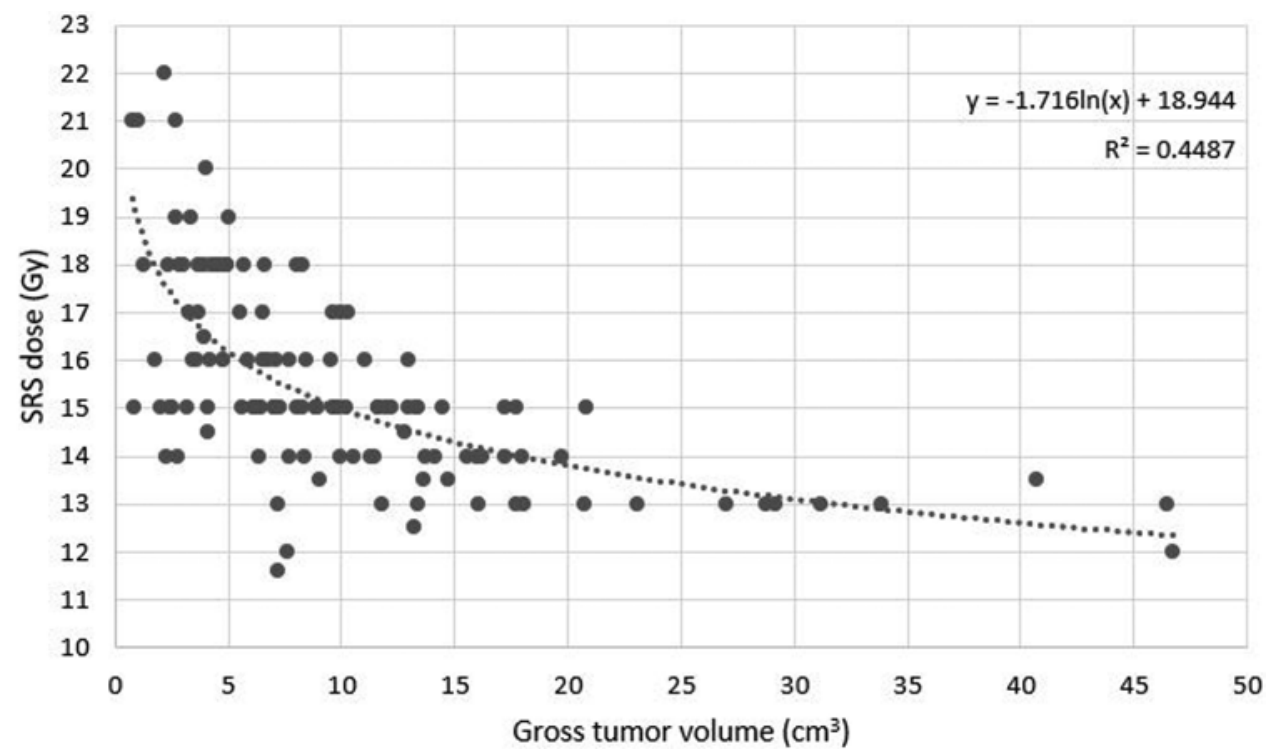

FIG. 1. SRS dose versus gross tumor volume. The best-fit regression was logarithmic. $R^{2}=$ coefficient of determination.

was death from any cause. OS event rates were estimated using the Kaplan-Meier product limit method and patients were censored at time that they were last known alive. Multivariable analysis for OS was performed using the Cox method. No variable selection algorithms were used in the multivariable analysis, as the goal was to determine the prognostic significance of known prognostic variables in the preoperative SRS setting. Multivariable analysis for intracranial outcomes included graded prognostic index (GPA), ${ }^{25}$ GTV volume, primary tumor site, systemic disease status (active defined as newly diagnosed or evidence of progression within the previous 3 months), SRS dose, and resection status (gross vs subtotal resection). The same variables were included in the OS multivariable analysis, except for the addition of age. All analyses were carried out using SAS version 9.4.0 statistical software (SAS Institute, Inc.). Significance testing was 2-sided, with $\mathrm{p}$ values $\leq 0.05$ considered statistically significant.

\section{Results}

\section{Patient and Treatment Characteristics}

A total of 120 patients with 128 treated lesions were initially eligible for the study. Two patients $(1.7 \%)$ with 2 lesions were treated with preoperative SRS but did not undergo surgery due to unrelated intervertebral disc infection in one case and unrelated complications of previous lumbar dural biopsy in the other. One patient was treated with preoperative SRS, but had nonmetastatic disease on pathologic review. That patient presented with headaches and a single $3-\mathrm{cm}$ left parietal lesion 3 years after radiographic stabilization of known metastatic neuroendocrine carcinoma. He subsequently underwent preoperative SRS and resection, and histological analysis revealed glioblastoma multiforme. These 3 patients were excluded, which left 117 patients with 125 treated lesions eligible for analysis. This group of 117 patients included an initial 24 patients who were enrolled in a prospective trial and an additional 93 patients whose cases were retrospectively reviewed (Table 1). The median total number of brain metastases was 1 (interquartile range [IQR] 1-2), the median GPA was 2.5 (IQR 2-3), and the median patient age was 58 years (IQR 51-68 years). Non-small cell lung cancer (NSCLC) was the most common primary tumor (42.7\%), followed by breast cancer (18.8\%), melanoma (15.4\%), and renal cell carcinoma (RCC, 11.1\%). GTR of the brain metastases was achieved in $95.2 \%$ of cases. The median interval between preoperative SRS and surgery was 2 days (IQR 1-4 days), the median index lesion GTV was $8.3 \mathrm{~cm}^{3}$ (IQR 4.6-13.3 $\mathrm{cm}^{3}$ ), and the median SRS dose was $15 \mathrm{~Gy}$ (IQR 14-17 Gy). The median cranial imaging follow-up period was 10.5 months for all patients and 18.7 months for alive patients. A total of 19 patients $(16.2 \%)$ were considered lost to cranial imaging follow-up, defined as no evidence of death and $\geq 6$ months since the last cranial imaging. The median imaging and clinical follow-up periods for patients lost to follow-up were 11.7 months and 23.4 months, respectively. See Fig. 1 for preoperative SRS dose versus GTV volume.

\section{Cavity Local Recurrence}

Overall, LR was identified in 26 cavities, for a crude rate of $20.8 \%$. The 1- and 2-year cavity LR rates were $19.9 \%$ and $25.1 \%$, respectively. The 1- and 2-year death without cavity LR rates were $25 \%$ and $39.9 \%$, respectively (Fig. 2). Of the $26 \mathrm{LR}$ events, 18 were treated with LR salvage therapy, consisting of WBRT $(n=7)$, partial brain radiation therapy $(\mathrm{RT})$ only $(\mathrm{n}=4)$, surgery and partial brain RT $(n=3)$, surgery only $(n=2)$, and systemic therapy only $(n=2)$. Index lesion GTV and SRS dose were not associated with risk of cavity LR in either univariable or multivariable analyses. Resection status was significantly associated with LR in univariable analysis (GTR vs STR [subtotal resection], hazard ratio [HR] 0.11, $\mathrm{p}=0.003)$. Of the 6 cavities in which STR had been performed, 4 were found to have LR at a median interval of 2.1 months after 
TABLE 1. Patient, tumor, and treatment characteristics

\begin{tabular}{|c|c|}
\hline Characteristic & Value \\
\hline No. of patients & $117(100 \%)$ \\
\hline No. of lesions & $125(100 \%)$ \\
\hline \multicolumn{2}{|l|}{ Sex } \\
\hline Male & $57(48.7 \%)$ \\
\hline Female & $60(51.3 \%)$ \\
\hline \multicolumn{2}{|l|}{ Race } \\
\hline White & $91(77.8 \%)$ \\
\hline Black & $22(18.8 \%)$ \\
\hline Other & $3(2.6 \%)$ \\
\hline Unknown & $1(1 \%)$ \\
\hline \multicolumn{2}{|l|}{ No. of brain metastases } \\
\hline 1 & $82(70.1 \%)$ \\
\hline 2 & $24(20.5 \%)$ \\
\hline 3 & $9(7.7 \%)$ \\
\hline 4 & $2(1.7 \%)$ \\
\hline \multicolumn{2}{|l|}{ Active systemic disease } \\
\hline Yes & $67(57.3 \%)$ \\
\hline No & $50(42.7 \%)$ \\
\hline \multicolumn{2}{|l|}{ Zubrod performance status } \\
\hline 0 & $51(43.6 \%)$ \\
\hline 1 & $45(38.5 \%)$ \\
\hline 2 & $18(15.4 \%)$ \\
\hline 3 & $3(2.6 \%)$ \\
\hline \multicolumn{2}{|l|}{ RPA class } \\
\hline 1 & $19(16.2 \%)$ \\
\hline 2 & $95(81.2 \%)$ \\
\hline 3 & $3(2.6 \%)$ \\
\hline \multicolumn{2}{|l|}{ GPA } \\
\hline $0.5-1$ & $5(4.3 \%)$ \\
\hline $1.5-2$ & $40(34.2 \%)$ \\
\hline $2.5-3$ & $54(46.2 \%)$ \\
\hline $3.5-4$ & $18(15.4 \%)$ \\
\hline \multicolumn{2}{|l|}{ Primary cancer } \\
\hline NSCLC & $50(42.7 \%)$ \\
\hline Breast & $22(18.8 \%)$ \\
\hline Melanoma & $18(15.4 \%)$ \\
\hline $\mathrm{RCC}$ & $13(11.1 \%)$ \\
\hline Gastrointestinal & $6(5.1 \%)$ \\
\hline Other & $8(6.8 \%)$ \\
\hline \multicolumn{2}{|l|}{ NSCLC subtype $(n=50)$} \\
\hline Squamous cell & $5(10 \%)$ \\
\hline Adenocarcinoma & $32(64 \%)$ \\
\hline Large cell, NOS & $9(18 \%)$ \\
\hline Unknown & $4(8 \%)$ \\
\hline \multicolumn{2}{|c|}{ Breast cancer ER status ( $n=22)$} \\
\hline Positive & $5(68.2 \%)$ \\
\hline Negative & $6(27.3 \%)$ \\
\hline Unknown & $1(4.5 \%)$ \\
\hline \multicolumn{2}{|c|}{ Breast cancer HER-2 status $(n=22)$} \\
\hline Positive & $10(45.5 \%)$ \\
\hline Negative & $8(36.4 \%)$ \\
\hline Unknown & $4(18.2 \%)$ \\
\hline \multicolumn{2}{|l|}{ Extent of resection } \\
\hline Gross total & $119(95.2 \%)$ \\
\hline Subtotal & $6(4.8 \%)$ \\
\hline
\end{tabular}

CONTINUED IN NEXT COLUMN »
» CONTINUED FROM PREVIOUS COLUMN

TABLE 1. Patient, tumor, and treatment characteristics

\begin{tabular}{lc}
\hline \multicolumn{1}{c}{ Characteristic } & Value \\
\hline Brain metastasis location & $48(38.4 \%)$ \\
Frontal & $28(22.4 \%)$ \\
Parietal & $19(15.2 \%)$ \\
Temporal & $7(5.6 \%)$ \\
Occipital & $21(16.8 \%)$ \\
Cerebellum & $2(1.6 \%)$ \\
Other & $58(51-68)$ \\
\hline Age in yrs, median (IQR) & $80 \%(80-80 \%)$ \\
\hline Prescription isodose line, median (IQR) & $1.46(1.36-1.54)$ \\
\hline Conformality index, median (IQR) & $15(14-17)$ \\
\hline SRS dose in Gy, median (IQR) & $8.3(4.6-13.3)$ \\
\hline Gross tumor vol in $\mathrm{cm}^{3}$ (IQR)
\end{tabular}

NOS = not otherwise specified; ER = estrogen receptor; HER-2 = human epidermal growth factor receptor 2 .

surgery. One patient died without cavity LR 10.3 months after surgery, and 1 patient was alive without recurrence 7.3 months after surgery but was lost to follow-up. The 6-month cavity LR rates for GTR and STR were $6.5 \%$ and $66.7 \%$, respectively. In MVA, resection status (GTR vs STR, HR 0.15, p = 0.008) and RCC primary (vs NSCLC, HR $0.1, p<0.001$ ) were significant predictors of cavity LR (Table 2).

\section{Distant Brain Failure and Use of Salvage WBRT}

Overall, 65 patients (55.6\%) experienced DBF. The 1and 2-year DBF rates were $45.3 \%$ and $60.2 \%$, respectively (Fig. 2). The 1- and 2-year rates of death without DBF were $16 \%$ and $23.3 \%$, respectively. Of the 65 patients who experienced DBF, $57(87.7 \%)$ received salvage therapy consist-

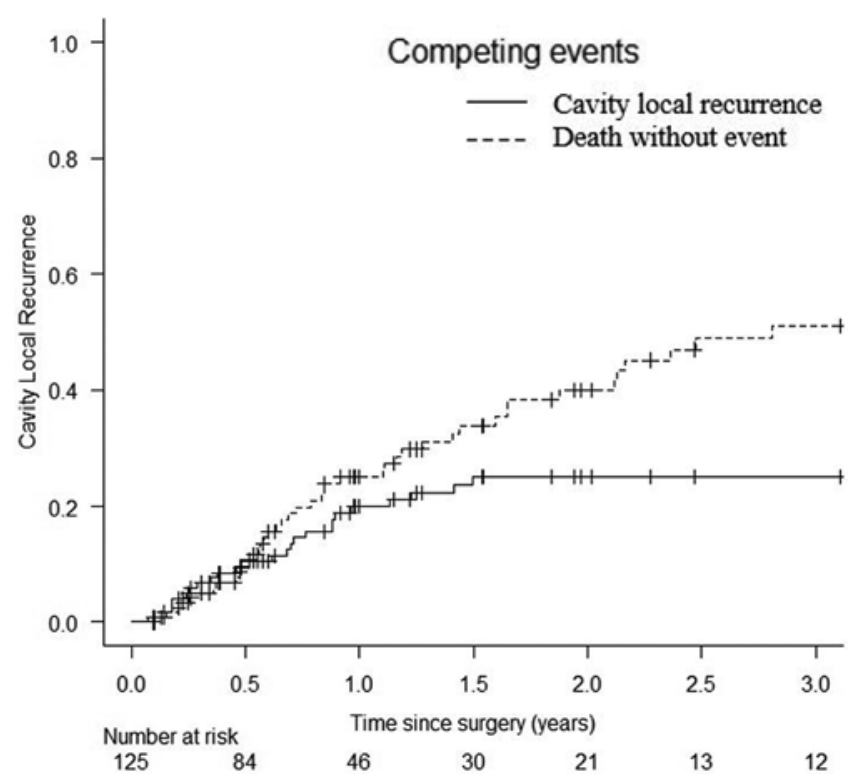

FIG. 2. Cumulative incidence of cavity local recurrence with competing risk of death. Curve truncated at 3 years. 
TABLE 2. Multivariable analysis of cavity local recurrence

\begin{tabular}{llcc}
\hline \multicolumn{1}{c}{ Variable } & $\mathrm{HR}$ & $95 \% \mathrm{Cl}$ & $\mathrm{p}$ Value \\
\hline Active systemic disease & & & \\
$\quad$ No & Ref & & \\
Yes & 0.44 & $0.16-1.17$ & 0.1 \\
\hline SRS dose & 0.91 & $0.7-1.18$ & 0.48 \\
\hline GPA & 0.99 & $0.48-2.05$ & 0.98 \\
\hline GTV (quartiles) & 1.2 & $0.8-1.81$ & 0.37 \\
\hline Resection status & & & \\
Gross total & 0.15 & $0.04-0.58$ & 0.008 \\
Subtotal & Ref & & \\
\hline Primary site or tumor type & & & \\
NSCLC & Ref & & \\
Breast & 1.28 & $0.52-3.16$ & 0.6 \\
Melanoma & 0.21 & $0.03-1.68$ & 0.14 \\
RCC & 0.1 & $0.02-0.16$ & $<0.001$ \\
Gastrointestinal & 2.23 & $0.39-1.27$ & 0.37 \\
Other & 1.79 & $0.62-5.21$ & 0.29 \\
\hline
\end{tabular}

Boldface type indicates statistical significance.

ing primarily of SRS alone $(\mathrm{n}=27)$ or WBRT $(\mathrm{n}=24)$. A total of 27 patients $(23.1 \%)$ received salvage WBRT. The 1- and 2-year salvage WBRT rates were $17.8 \%$ and $22.9 \%$, respectively. The 1- and 2-year rates of death without salvage WBRT were $24 \%$ and $45 \%$, respectively.

\section{Leptomeningeal Disease Recurrence}

A total of 6 patients $(5.1 \%)$ experienced LMD recurrence at a median of 11.6 months after surgery. The 1- and 2-year LMD rates were $4.3 \%$ and $4.3 \%$, respectively (Supplemental Fig. 1). The 1- and 2-year rates of death without LMD were $30 \%$ and $51.4 \%$, respectively. Of the 5 patients with STR, 1 (20\%) experienced LMD recurrence. There were not enough LMD events to perform further analyses.

\section{Radiation Necrosis and Other Toxicities}

Radiation necrosis (RN) was identified in a total of 11 cavities RN (8.8\%). In 5 cases, the RN was symptomatic and required treatment (45.5\% of RN events, $4 \%$ of entire cohort). Four symptomatic RN events were treated with steroids, while 1 required steroid treatment and resection. The 1- and 2-year RN rates were $5.1 \%$ and $8.1 \%$, respectively. The 1- and 2-year symptomatic RN rates were $2.6 \%$ and $4.8 \%$, respectively. Three patients $(2.6 \%)$ experienced grade 3 toxicity: symptomatic $\mathrm{RN}$ requiring surgery $(\mathrm{n}=$ 1), postoperative wound complications $(\mathrm{n}=1)$, and cavity hematoma requiring evacuation $(n=1)$.

\section{Composite Endpoint of Cavity LR, LMD, or Symptomatic RN}

Since the purpose of SRS as an adjunct to surgery is to maximize cavity local control while minimizing toxicity in the palliative setting, we combined cavity LR, symptomatic RN, and LMD recurrence into a composite endpoint to better assess overall toxicity and tumor control, consistent with our previous study. ${ }^{20}$ The 1- and 2-year composite endpoint rates were $24.5 \%$ and $30.7 \%$, respectively (Fig. 3), which are numerically similar to the 1- and

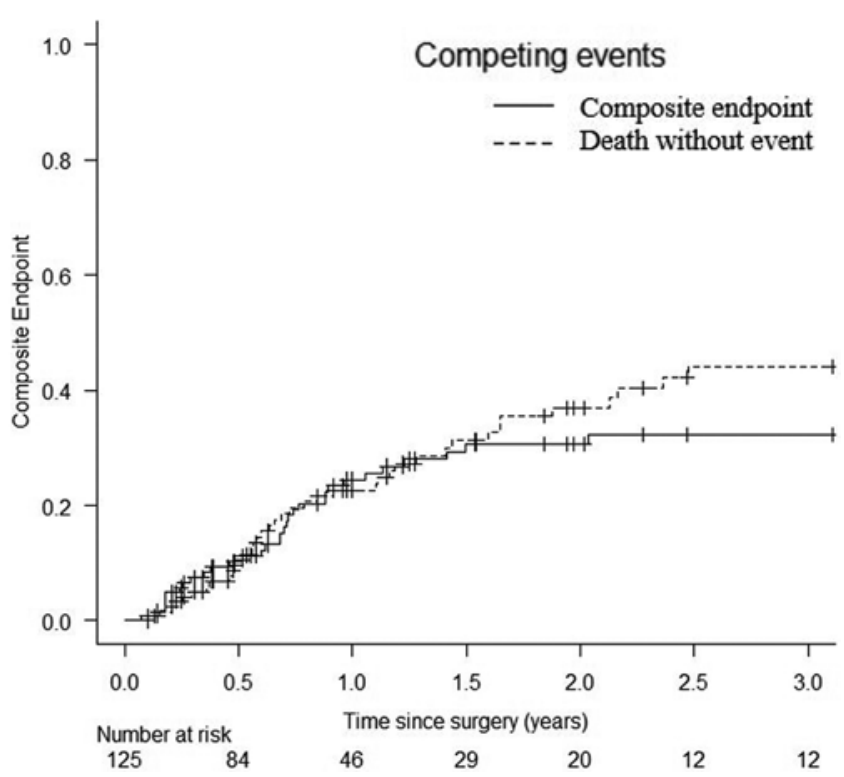

FIG. 3. Cumulative incidence of the composite endpoint of cavity local recurrence, leptomeningeal disease recurrence, or symptomatic radiation necrosis with competing risk of death. Curve truncated at 3 years.

2-year composite endpoint rates for the previously published earlier cohort of 66 patients treated with preoperative SRS of $15.8 \%$ and $27.9 \%$, respectively. ${ }^{20}$ The current composite endpoint incidence also compares favorably to the previously reported 1- and 2-year rates in 114 patients treated with postoperative SRS (31.8\% and 39.3\%, respectively). ${ }^{20}$ The 1- and 2-year death rates without the composite endpoint rate were $22.7 \%$ and $37 \%$, respectively. MVA demonstrated that resection status (GTR vs STR, HR $0.08, \mathrm{p}<0.001$ ) and RCC primary (vs NSCLC, HR $0.14, p<0.001)$ were significant predictors for the composite endpoint.

\section{Overall Survival}

The median clinical follow-up period was 14.9 months for all patients and 21.3 months for alive patients. A total of 89 patients $(76.1 \%)$ died. The median, 1-, and 2-year OS rates were 17.2 months (95\% confidence interval [CI] 12.3-22.1 months), 60.6\%, and 36.7\%, respectively (Supplemental Fig. 2). MVA for OS demonstrated that resection status (GTR vs STR, HR 0.38, p = 0.05), GPA (HR $0.54, p=0.02$ ), and breast cancer primary (vs NSCLC, HR $0.55, p=0.06$ ), were predictors of OS (Table 3). The median OS for patients with STR $(\mathrm{n}=5)$ was 8 months $(95 \%$ CI 2-14 months) versus 18.4 months (95\% CI 12.4-24.5 months) for GTR $(n=112, p=0.001)$.

\section{Discussion}

The original publication from our group reporting clinical outcomes after preoperative SRS included 47 patients, ${ }^{2}$ and subsequent published studies comparing preoperative SRS with postoperative SRS and postoperative WBRT, respectively, included 66 patients treated with preoperative SRS. ${ }^{20,21}$ This updated and expanded cohort with significantly more patients and longer cranial imag- 
TABLE 3. Multivariable analysis of overall survival

\begin{tabular}{llll}
\hline \multicolumn{1}{c}{ Variable } & HR & $95 \% \mathrm{Cl}$ & $\mathrm{p} \mathrm{Value}$ \\
\hline $\begin{array}{l}\text { Active systemic disease } \\
\text { No }\end{array}$ & Ref & & \\
$\quad$ Yes & 1.04 & $0.57-1.89$ & 0.9 \\
\hline SRS dose & 0.91 & $0.78-1.06$ & 0.21 \\
\hline GPA & 0.54 & $0.33-0.89$ & 0.02 \\
\hline GTV (quartiles) & 1.01 & $0.77-1.31$ & 0.95 \\
\hline Resection status & & & \\
$\quad$ Gross total & 0.38 & $0.14-0.99$ & 0.05 \\
$\quad$ Subtotal & Ref & & \\
Primary site or tumor type & & & \\
$\quad$ NSCLC & Ref & & \\
Breast & 0.55 & $0.29-1.03$ & 0.06 \\
$\quad$ Melanoma & 0.52 & $0.25-1.08$ & 0.08 \\
RCC & 0.62 & $0.3-1.25$ & 0.18 \\
$\quad$ Gastrointestinal & 1.9 & $0.72-5.04$ & 0.2 \\
$\quad$ Other & 1.07 & $0.42-2.71$ & 0.89 \\
\hline Age in yrs & 1 & $0.98-1.02$ & 0.6 \\
\hline
\end{tabular}

Boldface type indicates statistical significance.

ing and clinical follow-up periods confirms these previous results and demonstrates that the preoperative SRS paradigm continues to be associated with excellent cavity local control and notably low risk of RN or LMD recurrence.

Preoperative SRS has several potential advantages compared to postoperative SRS, which provide the rationale for its use. Preoperative SRS treats the intact tumor volume, which is well defined, readily identifiable on imaging, and does not require any margin expansion for target delineation uncertainty. Additionally, preoperative SRS is given prior to surgery, with the potential advantage of increased compliance, given the variable postoperative clinical course for patients and the variable timing of postoperative SRS due to the need for healing and surgical recovery. ${ }^{6,12}$ Lastly, preoperative SRS may be associated with reduced risk of LMD due to sterilization of tumor cells prior to spillage at the time of surgery. ${ }^{20}$

We used an approximate 10-20\% dose reduction scheme for preoperative SRS treatments, with doses as low as 12 Gy. There are several reasons for this reduced dosing. First, the goal of preoperative SRS is to control microscopic disease, given that the brain metastasis will be resected. Since reduced tumor burden (with resection) likely requires reduced RT dose for the same control, the dose was reduced. Second, treatment of larger volumes with single-fraction SRS at standard dosing is associated with higher risk of RN compared with treatment of smaller volumes or hypofractionated SRS..$^{10,17}$ Preoperative SRS is ideally completed in a single fraction in order to avoid the need to delay surgery, as would be the case if the SRS was delivered over 3-5 fractions. This dose reduction allows for single-fraction SRS to larger volumes without excessive RN risk. The use of 10-20\% dose-reduced preoperative SRS has been now been validated with clinical outcomes demonstrating excellent cavity local control with low risk of RN in the GTR setting.

However, a notable new finding from this analysis is the significant detriment in cavity LR and OS seen for patients who underwent STR after preoperative SRS. All patients in our prior preoperative SRS studies had undergone GTR. Although the number of cases involved was small $(n=6$ cavities) and the findings should therefore be interpreted with caution, STR was associated with an over 6-fold increased risk of cavity progression and an over 2-fold increased risk of death relative to GTR in MVA. No patient who underwent STR received planned adjuvant RT after surgery. This high rate of LR suggests that dose-reduced preoperative SRS is not able to control residual gross disease. However, other factors, such as tumor location, tumor shape or composition (cystic vs solid), or presence of hemorrhage may have influenced both the extent of resection and risk of subsequent LR. We were unable to examine this in more detail due to the small number of patients with STR. However, based on these data, we would not recommend preoperative SRS for patients who are likely to undergo STR as determined by the neurosurgeon preoperatively. We would also recommend considering reresection if feasible to achieve a GTR or additional RT in the setting of unexpected STR to maximize local control, although the ideal regimen, efficacy, and toxicity of additional RT after preoperative SRS is not yet known and will be the subject of future studies. Proper patient selection to avoid STR is important and is generally achievable, as evidenced by the fact that $95 \%$ of index brain metastases in this study did undergo GTR.

Another important finding from this study is that in the setting of GTR, larger tumor size and lower SRS dose were not associated with increased risk of LR. This is in contrast to the findings of 2 prospective studies of postoperative SRS, in which larger preoperative tumor diameter was a significant negative prognostic factor for increased cavity LR. ${ }^{6,16}$ Additionally, although a theoretical concern for preoperative SRS is the potential inadvertent treatment of nonmetastatic disease when SRS is given prior to pathologic analysis, only 1 patient $(0.8 \%)$ who received preoperative SRS had nonmetastatic disease (the patient had known stable metastatic neuroendocrine cancer and a new 3-cm left parietal lesion treated with preoperative SRS and subsequently found to be glioblastoma).

Our group previously demonstrated in a multi-institutional retrospective study of preoperative versus postoperative SRS that there was no difference between groups with respect to $\mathrm{OSq}$, cavity LR, or DBF. ${ }^{20}$ The univariate 1 -year rate of cavity LR was $15.9 \%$ versus $12.6 \%(p=0.33$ ) for preoperative versus postoperative SRS. However, preoperative SRS had a significantly lower rate of LMD recurrence compared with postoperative SRS, with a 2-year rate of $3.2 \%$ versus $16.6 \%(p=0.01)$. Similar results were found for RN and symptomatic RN, with a 2-year rate of symptomatic RN of $4.9 \%$ for preoperative SRS versus $16.4 \%$ for postoperative SRS $(p=0.01)$. The estimated event rates from this updated study are qualitatively on par with those previously reported for the earlier 66-patient cohort treated with preoperative SRS: 1-year LR, 19.9\% vs $15.9 \%$; 2-year LMD, $4.3 \%$ vs 3.2\%; 2-year symptomatic RN, $4.8 \%$ vs $4.9 \%$; 2 -year composite endpoint, $30.7 \%$ vs $27.9 \%$, respectively) indicating no sign of worse efficacy or increased toxicity with more patient-years analyzed. 
To the best of our knowledge, there is only 1 other published study reporting clinical outcomes for preoperative SRS. ${ }^{26}$ This is a retrospective study of 19 cases, including 8 in which the patients were treated for local disease recurrence after previous index brain metastasis treatment (with the index treatment having been SRS in 5 cases and surgery alone in 3). The median duration of follow-up was 6.3 months, but it was not stated whether this referred to cranial imaging or clinical follow-up. Two patients experienced cavity LR, 1 patient experienced LMD, and 1 patient experienced RN (but this patient had received previous SRS to the same site).

The potential for selection bias is the primary limitation of this study due to its largely retrospective nature and the fact that all patients were treated at a single institution. In addition, there is a lack of neurocognitive, neurological death, and quality-of-life information for the cohort. The study strengths include its homogeneous treatment paradigm and follow-up/surveillance schedules, comparatively large patient numbers, and use of cumulative incidence with competing risk statistical methodology. This method has been shown to more accurately estimate event rates in the setting where death without the event can be common, such as in the treatment of patients with brain metastases. ${ }^{4,5}$

\section{Conclusions}

This expanded and updated analysis confirms that single-fraction preoperative SRS confers excellent cavity local control with very low risk of RN or LMD. Preoperative SRS has several potential advantages compared to postoperative SRS, including reduced risk of RN due to smaller irradiated volume without need for cavity margin expansion and reduced risk of LMD due to sterilization of tumor cells prior to spillage at the time of surgery. Subtotal resection, although infrequent in this study, is associated with significantly worse cavity LR and OS. Preoperative SRS should be avoided in cases in which STR is likely. Based on these results, a cooperative group randomized trial of preoperative versus postoperative SRS is being designed at this time.

\section{References}

1. Aoyama H, Shirato H, Tago M, Nakagawa K, Toyoda T, Hatano K, et al: Stereotactic radiosurgery plus whole-brain radiation therapy vs stereotactic radiosurgery alone for treatment of brain metastases: a randomized controlled trial. JAMA 295:2483-2491, 2006

2. Asher AL, Burri SH, Wiggins WF, Kelly RP, Boltes MO, Mehrlich M, et al: A new treatment paradigm: neoadjuvant radiosurgery before surgical resection of brain metastases with analysis of local tumor recurrence. Int J Radiat Oncol Biol Phys 88:899-906, 2014

3. ASTRO: ASTRO releases second list of 5 radiation oncology treatments to question as part of national Choosing Wisely campaign. Choosing Wisely. September 15, 2014. (http:// www.choosingwisely.org/astro-releases-second-list/) [Accessed August 28, 2018]

4. Austin PC, Lee DS, Fine JP: Introduction to the analysis of survival data in the presence of competing risks. Circulation 133:601-609, 2016

5. Berry SD, Ngo L, Samelson EJ, Kiel DP: Competing risk of death: an important consideration in studies of older adults. J Am Geriatr Soc 58:783-787, 2010

6. Brennan C, Yang TJ, Hilden P, Zhang Z, Chan K, Yamada Y, et al: A phase 2 trial of stereotactic radiosurgery boost after surgical resection for brain metastases. Int J Radiat Oncol Biol Phys 88:130-136, 2014

7. Brown PD, Ballman KV, Cerhan JH, Anderson SK, Carrero $\mathrm{XW}$, Whitton AC, et al: Postoperative stereotactic radiosurgery compared with whole brain radiotherapy for resected metastatic brain disease (NCCTG N107C/CEC·3): a multicentre, randomised, controlled, phase 3 trial. Lancet Oncol 18:1049-1060, 2017

8. Brown PD, Jaeckle K, Ballman KV, Farace E, Cerhan JH, Anderson SK, et al: Effect of radiosurgery alone vs radiosurgery with whole brain radiation therapy on cognitive function in patients with 1 to 3 brain metastases: a randomized clinical trial. JAMA 316:401-409, 2016

9. Chang EL, Wefel JS, Hess KR, Allen PK, Lang FF, Kornguth DG, et al: Neurocognition in patients with brain metastases treated with radiosurgery or radiosurgery plus whole-brain irradiation: a randomised controlled trial. Lancet Oncol 10:1037-1044, 2009

10. Eaton BR, LaRiviere MJ, Kim S, Prabhu RS, Patel K, Kandula $\mathrm{S}$, et al: Hypofractionated radiosurgery has a better safety profile than single fraction radiosurgery for large resected brain metastases. J Neurooncol 123:103-111, 2015 (Erratum in J Neurooncol 123:113, 2015)

11. Fine JP, Gray RJ: A proportional hazards model for the subdistribution of a competing risk. JAMA 94:496-509, 1999

12. Jarvis LA, Simmons NE, Bellerive M, Erkmen K, Eskey CJ, Gladstone DJ, et al: Tumor bed dynamics after surgical resection of brain metastases: implications for postoperative radiosurgery. Int J Radiat Oncol Biol Phys 84:943-948, 2012

13. Johnson MD, Avkshtol V, Baschnagel AM, Meyer K, Ye H, Grills IS, et al: Surgical resection of brain metastases and the risk of leptomeningeal recurrence in patients treated with stereotactic radiosurgery. Int J Radiat Oncol Biol Phys 94:537-543, 2016

14. Kocher M, Soffietti R, Abacioglu U, Villà S, Fauchon F, Baumert BG, et al: Adjuvant whole-brain radiotherapy versus observation after radiosurgery or surgical resection of one to three cerebral metastases: results of the EORTC 2295226001 study. J Clin Oncol 29:134-141, 2011

15. Kumar AJ, Leeds NE, Fuller GN, Van Tassel P, Maor MH, Sawaya RE, et al: Malignant gliomas: MR imaging spectrum of radiation therapy- and chemotherapy-induced necrosis of the brain after treatment. Radiology 217:377-384, 2000

16. Mahajan A, Ahmed S, McAleer MF, Weinberg JS, Li J, Brown P, et al: Post-operative stereotactic radiosurgery versus observation for completely resected brain metastases: a single-centre, randomised, controlled, phase 3 trial. Lancet Oncol 18:1040-1048, 2017

17. Minniti G, Scaringi C, Paolini S, Lanzetta G, Romano A, Cicone F, et al: Single-fraction versus multifraction $(3 \times 9$ Gy) stereotactic radiosurgery for large $(>2 \mathrm{~cm})$ brain metastases: a comparative analysis of local control and risk of radiation-induced brain necrosis. Int J Radiat Oncol Biol Phys 95:1142-1148, 2016

18. Nayak L, Lee EQ, Wen PY: Epidemiology of brain metastases. Curr Oncol Rep 14:48-54, 2012

19. Patchell RA, Tibbs PA, Regine WF, Dempsey RJ, Mohiuddin $\mathrm{M}$, Kryscio RJ, et al: Postoperative radiotherapy in the treatment of single metastases to the brain: a randomized trial. JAMA 280:1485-1489, 1998

20. Patel KR, Burri SH, Asher AL, Crocker IR, Fraser RW, Zhang $\mathrm{C}$, et al: Comparing preoperative with postoperative stereotactic radiosurgery for resectable brain metastases: a multi-institutional analysis. Neurosurgery 79:279-285, 2016

21. Patel KR, Burri SH, Boselli D, Symanowski JT, Asher AL, 
Sumrall A, et al: Comparing pre-operative stereotactic radiosurgery (SRS) to post-operative whole brain radiation therapy (WBRT) for resectable brain metastases: a multi-institutional analysis. J Neurooncol 131:611-618, 2017

22. Shaw E, Scott C, Souhami L, Dinapoli R, Kline R, Loeffler J, et al: Single dose radiosurgical treatment of recurrent previously irradiated primary brain tumors and brain metastases: final report of RTOG protocol 90-05. Int J Radiat Oncol Biol Phys 47:291-298, 2000

23. Smedby KE, Brandt L, Bäcklund ML, Blomqvist P: Brain metastases admissions in Sweden between 1987 and 2006. Br J Cancer 101:1919-1924, 2009

24. Soffietti R, Kocher M, Abacioglu UM, Villa S, Fauchon F, Baumert BG, et al: A European Organisation for Research and Treatment of Cancer phase III trial of adjuvant wholebrain radiotherapy versus observation in patients with one to three brain metastases from solid tumors after surgical resection or radiosurgery: quality-of-life results. J Clin Oncol 31:65-72, 2013

25. Sperduto PW, Berkey B, Gaspar LE, Mehta M, Curran W: A new prognostic index and comparison to three other indices for patients with brain metastases: an analysis of 1,960 patients in the RTOG database. Int J Radiat Oncol Biol Phys 70:510-514, 2008

26. Vetlova E, Golbin DA, Golanov AV, Potapov AA, Banov SM, Antipina N, et al: Preoperative stereotactic radiosurgery of brain metastases: preliminary results. Cureus 9:e1987, 2017

\section{Disclosures}

Dr. Ward reports receipt of financial compensation (paid to
Southeast Radiation Oncology Group) for participation on the AstraZeneca Advisory Board.

\section{Author Contributions}

Conception and design: Prabhu, Asher, Patel, Burri. Acquisition of data: Prabhu, Miller, Heinzerling, Moeller, Lankford, McCammon, Fasola, Patel, Press, Sumrall, Ward. Analysis and interpretation of data: Prabhu, Asher, Burri. Drafting the article: Prabhu. Critically revising the article: all authors. Reviewed submitted version of manuscript: all authors. Approved the final version of the manuscript on behalf of all authors: Prabhu. Statistical analysis: Prabhu. Administrative/technical/material support: Miller, Burri. Study supervision: Prabhu, Burri.

\section{Supplemental Information}

\section{Online-Only Content}

Supplemental material is available with the online version of the article.

Supplemental Figs. 1 and 2. https://thejns.org/doi/suppl/10. 3171/2018.7.JNS181293.

\section{Correspondence}

Roshan S. Prabhu: Southeast Radiation Oncology Group, Levine Cancer Institute, Charlotte, NC. roshansprabhu@gmail.com. 